\title{
THE PATHOLOGICAL ANATOMY OF ACUTE LOCOMOTOR ATAXIA.
}

\author{
BY Dr, L, PUTZEL.
}

\section{THE following is a brief history of the case which forms the basis of my remarks this evening:}

J. D., at thirty-five years, a salesman, married ; entered Randall's Island Hospital on September 4, 1883 . The family history is unimportant. The patient has had gonorrhcea four times; gives no evidence of syphilis; habits regular, but he used to drink hard on holidays.

The present illness has lasted nine montlss; it began with ptosis of the right eye, the patient seeing double; shortly afterward he began to have shooting pains in the legs and arms. The pains were worse at night and before a storm. In a few weeks he had to quit work on account of the pains and weakness in lower limbs. Failure of appetite. About five months ago the patient got so weak that he was unable to walk, and had trouble in holding his water.

Present condition (Oct. 4, I883). Patient walks with assistance, but has a characteristic heel gait, and must watch the motions of his feet; he reels to and fro, when standing with eyes closed.

Anæsthesia and analgesia are present below Scarpa's triangle in both thighs and legs; the patellar reflexes are absent. Slight inco-ordination of upper limbs and some numbness of the fingers. Partial ptosis of right eye; both pupils very much dilated and react poorly to light ; optic papillæ normal ; special senses normal.

The dynamometer is forced to 40 by the right hand, to 36 by the left hand.

October 24 th. - Had a pulmonary hemorrlage ; dulness and a few moist râles over upper lobe of left lung.

The power of the lower limbs improved, but the inco-ordination rapidly grew worse, the limbs jerking in all directions upon at-

${ }^{1}$ Read before the Section on Neurology of the N. Y. Academy of Medicine May $8, \mathrm{r} 885$. 
tempting any voluntary movements. There is very marked emaciation of all the limbs, but the muscular power appears to be normal compared with the amount of muscular tissue retained. The ptosis disappeared almost entirely ; eyesight remained normal. 'The phthisis kept on advancing, and patient died January 7,1885 , without the development of any fresh symptoms on the part if the nervous system. The gross muscular power of the limbs remained good until within a short time of death.

The post-mortem examination revealed exquisite sclerosis of the posterior columns throughout their cntire extent, and what seemed to me to be a multiple sclerosis in various other parts of the cord. The gray matter appeared entirely normal.

Microscopic examination shows exquisite sclerosis of the posterior columns (enormous increase of the neuroglia and of nuclei), together with a general increase of the connective elements in the remaining portions of the cord, with the exception of the gray matter, which seems to be perfectly normal. In addition, the blood-vessels of the posterior columns are very much dilated, and their walls are exceedingly thickened, particularly the adventitia, in which the nuclei are enormously increased. In the anterior portions of the posterior columns, particularly in the vicinity of the posterior commissure, the nerve elements proper are very well preserved, despite the great proliferation of the neuroglia. The changes are also less marked in the posterior root zones. In addition, a number of intact nerve fibres can be seen in the more posterior portions of the posterior columns, and even very close to the pia mater. Under a high power these appear in no wise different from the fibres in other portions of the cord. The nuclei around the central canal are increascd in number, and thcre scems to be, also, an increase of fibrous tissue in this locality. Here and there in the other columns of the cord are seen bloodvessels which also present a considerable increase of nuclei.

If we take into consideration both the histological appearances and the clinical history of this case (the rapid loss of power and the acute onset of the ataxia, with the subsequent restoration of power to the normal), I think we are justified in concluding that the disease was primarily a dif... 
fuse myelitis, and that this inflammation cleared up in great measure in all other parts of the cord except the posterior columns. In these it continued to advance, and thus gave rise to the typical symptoms of locomotor ataxia.

According to the current theory of ataxia, the disease is primarily a degeneration of the axis-cylinders of the posterior columns, the increase of the neuroglia being regarded as secondary. For the majority of cases this theory, in my opinion, is well founded, and it is unnecessary for me to present the arguments usually advanced to support this view.

But during the last five or six years I have observed clinically quite a number of cases in which this theory did not appear to me to explain the clinical symptoms. These cases were all marked by their rapid onset and by the fact that, as in this case also, loss of power was a prominent initial factor-this symptom only clearing up as the ataxic symptoms became more prominent. The subsequent history presented no peculiarities.

I may here mention that I have under observation at the present time a case which seems to me to be similar in character to those referred to, but differs from them in the situation of the lesion. In this case, the primary symptoms for a long time were those of primary lateral sclerosis (rigidity of the limbs, greatly increased tendon reflexes, stiff gait), but after the lapse of a year or more, the symptoms of multiple spinal sclerosis have been super-added.

Very little attention has been paid by writers to the variety of ataxia under consideration, In fact I do not, at the present time, remember a single writer except Vulpian who has discussed the subject, though I must confess that, on account of lack of time, I have been unable to make a careful study of the literature of the question.

Vulpian merely mentions, in a few cursory remarks, that ataxia is sometimes secondary to other diseases of the cord, viz.: Pott's disease or compression of the cord from other causes, chronic spinal meningitis, meningo-myelitis, chronic pachymeningitis, descending secondary lateral leucomyelitis, etc. 
The subject seems to me to be an important one since, if we may indulge in a priori reasoning, this variety of ataxia is the only one which offers any hope of therapeutic relief. I must confess, however, that in my own experience thus far this hope has proved fruitless. 